\title{
AQUA2015: il Congresso IAH di Roma
}

Marco Petitta - Presidente del Comitato Italiano IAH - marco.petitta@uniroma1.it Daniela Ducci - Segretario del Comitato Italiano IAH - segretario@iahitaly.it

Come ogni anno dedichiamo questa rubrica all'annuale congresso Mondiale dell'IAH. Ma stavolta è assai diverso per molti motivi. E' stato il "nostro" Congresso, perché si è tenuto a Roma, ma non solo. Perché è stato costruito step by step in tre anni di duro lavoro. Perché è stato un immenso successo. Perché le keynote lectures sono state di alto livello. Perché gli interventi e i dibattiti scientifici sono stati interessanti e affollati. Perché si è mangiato benissimo. Perché tutti sono rimasti affascinati da Eataly. Ma soprattutto perché la partecipazione della comunità idrogeologica italiana è stata strepitosa, sia in termini quantitativi (circa 160 delegati) che qualitativi (174 lavori presentati e 30 convenors), come testimoniato anche dallo splendido documentario sull'Idrogeologia in Italia realizzato per il Congresso da A. Gargini (https:// www.aqua2015.com/video/Hydrogeoitaly.mp4). Insomma la maggiore soddisfazione risiede proprio nella forte crescita che ha fatto registrare l'idrogeologia in Italia e questo Congresso ha permesso di diffondere in tutto il mondo la nostra cultura idrogeologica. Non siamo più considerati di secondo piano, abbiamo dimostrato l'esistenza di una "scuola" idrogeologica italiana, formata non solo da accademici, ma anche dai tanti professionisti e aziende che operano all'avanguardia in vari ambiti e massicciamente presenti a Roma.

Ma veniamo al resoconto dettagliato: dal 13 al 18 Settembre, Roma ha accolto per il $42^{\text {esimo }}$ congresso internazionale della IAH, AQUA2015, un totale di oltre 800 partecipanti, tra cui circa 750 delegati e 33 accompagnatori, provenienti da 84 paesi, e più precisamente 322 dall'Europa (Italia esclusa), 127 dalle Americhe, 54 dall'Africa, 130 dall'Asia e 29 dall'Oceania. 25 delegati provenienti dai paesi emergenti e in via di sviluppo hanno potuto usufruire per l'iscrizione del contributo dell'UNESCO. Gli idrogeologi di tutto il mondo si sono confrontati sul tema "Back to the future", all'interno della Città Universitaria della Sapienza, in Aula Magna per le principali sessioni ed eventi (side event e workshop) e nelle aule del Dipartimento di Scienze della Terra, alcune delle quali appena

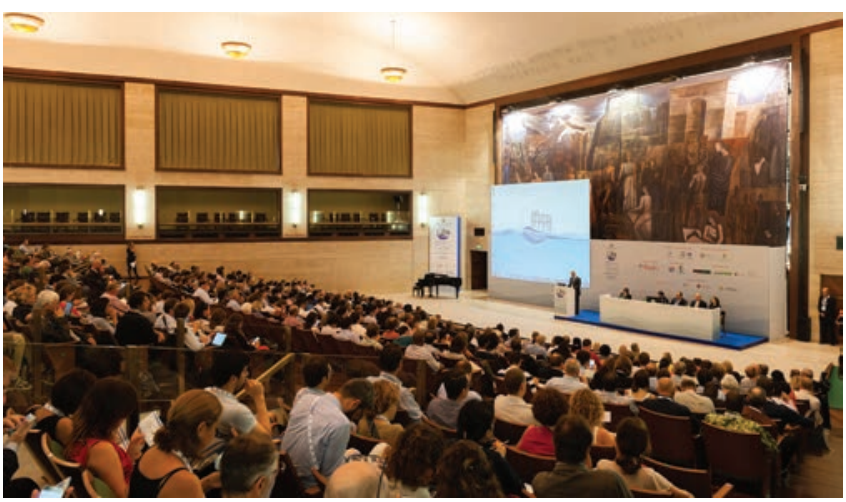

Fig. 1: La cerimonia inaugurale nell'Aula Magna della Sapienza. ristrutturate. In tutte le aule la connessione wireless, messa a punto per il congresso, ha funzionato egregiamente permettendo ai congressisti, sia di lavorare navigando in rete, che di collegarsi al sito www.aqua2015.com dove il programma scientifico consultabile on-line è stato aggiornato in tempo reale, e dove ad oggi è possibile per tutti i congressisti scaricare presentazioni e poster. La navigazione in rete e la consultazione del programma sono state anche facilitate, per chi non disponesse un proprio dispositivo, dal tablet 7" inserito come "gadget", nella bag del congresso, un simpatico e colorato zainetto, nel quale era anche contenuto il programma tascabile e vario materiale informativo. Il programma, come preannunciato, è stato ampio e ha richiesto in molti casi un attento studio (anche se la funzione "programma personalizzato" del sito è stata di grande aiuto). Sono state infatti attivate 7 sessioni orali parallele, che hanno coperto gli 8 temi principali con oltre 40 sessioni e più di 400 presentazioni, distribuite nell'arco della settimana, tutte estremamente gradite dai partecipanti. Le defezioni sono state pochissime e brillantemente risolte dai Convenors che hanno stimolato nei tempi morti interessanti discussioni. La sessione poster ha potuto contare su circa 350 poster elettronici, illustrati brevemente dagli Autori secondo un calendario prestabilito, ed è stata organizzata su 6 postazioni dotate di schermi da 55" e tablet di consultazione, da cui era possibile accedere on-demand a qualsiasi poster. Questa sessione ha presentato delle criticità dovuta non all'innovazione tecnologica degli e-poster, che hanno funzionato benissimo, ma all'eccessivo affollamento, causato da spazi troppo ristretti, e soprattutto da un clima caldissimo che è stato croce e delizia dei partecipanti. Fortunatamente gli e-poster erano e sono tuttora consultabili in rete sul sito.

Nell'Aula Magna si sono tenute delle emozionanti cerimonie inaugurali e finali, nonché l'Assemblea IAH, con annesse premiazioni, che hanno visto ancora protagonisti gli italiani: Alice Aureli dell'UNESCO ha ricevuto il premio per il suo impegno nella governance dell'acqua, Giovanni Barrocu è stato nominato Socio Onorario per la sua lunga e prestigiosa attività nella IAH, e Pasquale Steduto della FAO ha ricevuto il premio alla carriera. L'Early Career Hydrogeologist's Network ha assegnato, come sempre, i premi per la miglior presentazione ed il miglior poster presentati da giovani idrogeologi, come anche la Regional Groundwater Flow Commission con premi dedicati al proprio tema. Ma l'Aula Magna ha ospitato anche eventi culturali, tra cui ha spiccato la bellissima esibizione canora della soprano Di Tonno, nonché le 8 keynotes ognuna dedicata ad un diverso topic congressuale. Come invited speakers hanno infatti partecipato: Ramon Aravena (Department of Earth and Environmental Sciences, University of Waterloo, Ontario, Canada), Alice Aureli (UNESCO-IHP, Paris, France), John A. Cherry (University of Waterloo, ON, 


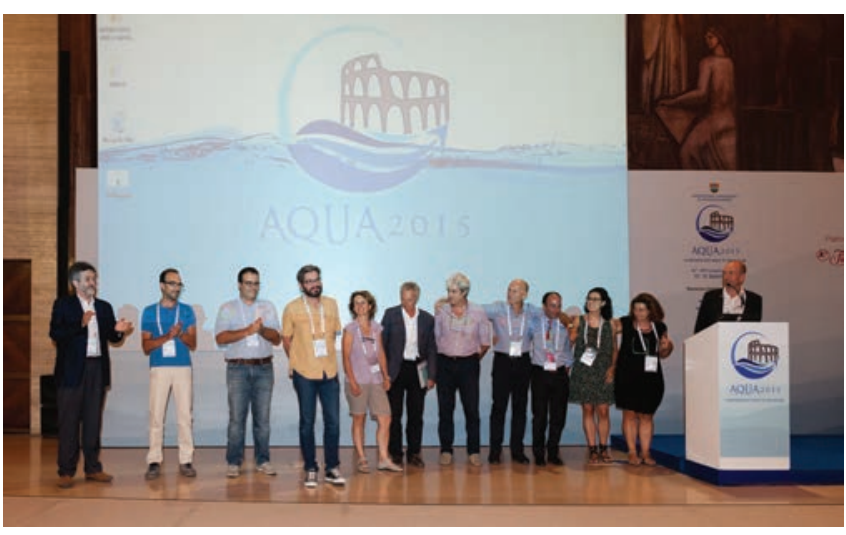

Fig. 2: Il comitato organizzatore durante la cerimonia di chiusura.

Canada), Ghislain De Marsily (University Paris VI, France), Nico Goldscheider (Karlsruhe Institute of Technology, Germany), la Darcy Lecture di Rainer Helmig (University of Stuttgart, Germany), Beth Parker (University of Guelph, Canada), Chunmiao Zheng (Peking University, China) e Clifford I. Voss (U.S. Geological Survey, USA). Tutti gli interventi a invito hanno consentito di apprezzare la attualità dei temi idrogeologici proposti per il congresso e di conoscere i nuovi approcci metodologici internazionali sui vari topic. Lalto livello degli interventi è dimostrato dall'ottimo successo di pubblico, ma ci preme segnalare la keynote tenuta da G. De Marsily, un mito della idrogeologia moderna, che nonostante non sia proprio un giovanotto, ha affrontato un tema modernissimo come l'uso sostenibile delle acque sotterranee con un approccio molto innovativo.

Sempre nell'Aula Magna si sono tenuti workshop tematici (side events), quali la presentazione del rapporto UNESCO WWAP sulle risorse idriche, quella sul tema della Governance dell'Acqua con UNESCO e FAO in prima linea, l'esposizione dei progetti europei KINDRA e FREEWAT di Horizon2020 dedicati al tema acqua e coordinati da due soci IAH italiani, la presentazione tenuta da Voss, curata da ECHN insieme all'Editorial Office di Hydrogeology Journal, l'interessante contributo di ESRI-ITALIA sulle piattaforme ArcGIS per le acque sotterranee e infine, nella giornata di venerdì, il workshop organizzato in collaborazione con ANIPA, sulla perforazione dei pozzi, al quale era possibile iscriversi anche separatamente. Hanno partecipato al singolo evento, che prevedeva anche riconoscimento dei crediti APC per i geologi, una trentina di professionisti operanti nel mondo delle acque sotterranee. Non vanno poi dimenticati i cinque corsi pre-congresso, che sono iniziati a partire dal giovedì prima del Congresso raccogliendo quasi 100 partecipanti in totale e si sono conclusi all'apertura del congresso domenica 13 , con l'evento gratuito tenuto da UNESCO GRAPHIC sui cambiamenti climatici. Il mercoledì 16 si sono tenute le uscite tecniche di metà settimana, e non possiamo tacere i complimenti che tutti i partecipanti hanno fatto all'organizzazione degli otto field trip: Roma Antica, i vulcani nord laziali, la foce del Tevere, i rilievi carsici dell'Appennino, la Piana Pontina, l'area termale di Tivoli, il Fucino e infine le sorgenti Ferrarelle a Riardo. Le guide dei field trips e le foto realizzate sono disponibili rispettivamente su https://www.aqua2015. com/img/field-trips/guides/ e su https:/www.aqua2015.com/ photos.php?day=16. Una cinquantina di congressisti e accompagnatori hanno invece preferito restare a Roma per assistere all'Udienza Papale, nel corso della quale il Pontefice ha anche citato il nostro Congresso.

Tutto noi del Comitato Organizzatore speriamo che il Congresso venga ricordato non solo per il livello scientifico raggiunto, ma anche per i numerosi eventi sociali, che hanno favorito la socializzazione, le discussioni e i contatti tra i Congressisti in modo informale e rilassato. Il consueto Welcome Cocktail si è tenuto nella terrazza dell'aula magna della Sapienza con un ottimo catering (stessa location e qualità per i lunch di tutte le giornate congressuali). L'inaspettata e forte pioggia (eh sì, sono stati proprio dei giorni a clima tropicale!) ha costretto, ma senza alcun rammarico, i congressisti a trattenersi ben oltre l'orario previsto avendo l'opportunità di continuare a brindare con dell'ottimo spumante e vino. Il lunedì sera si è tenuta la serata dedicata agli Early Career in un locale nel vicino quartiere di San Lorenzo, dove è stata la birra a farla da padrona, oltre alle originali competizioni idrogeologiche messe in campo dagli organizzatori. I più âgée hanno avuto modo di approfittare dell'invito per un emozionante spettacolo di luci e suoni ai Fori Imperiali da parte del Sindaco di Roma, che è intervenuto alla cerimonia inaugurale presentando la carta idrogeologica di Roma, distribuita nella borsa congressuale; Il martedì si è tenuta la cena nel centro storico e infine la cena sociale il giovedì presso EATALY, durante la quale oltre a mangiare ... e mangiare molto bene... si è giocato a lungo sul tema linguistico-idrogeologico-enologico.

Non ci resta che ringraziare i partecipanti, gli ospiti, i numerosi sponsor (la cui lista è disponibile sui due siti www. iah2015.org e www.aqua2015.com), gli studenti dello staff e i vari collaboratori della Sapienza, il personale della Triumph nostro partner organizzativo, $i$ colleghi che hanno guidato $i$ vari tour, ed infine tutti i componenti del Comitato Organizzatore. Siamo consapevoli che tutti hanno contribuito al successo dell'evento, per il quale stiamo continuando a ricevere complimenti dalla comunità idrogeologica internazionale.

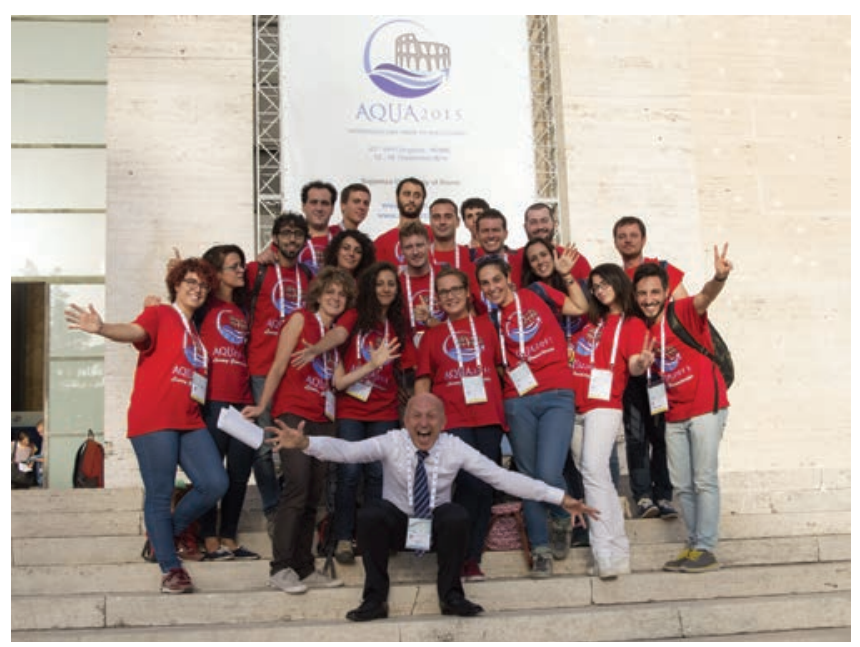

Fig. 3: Lo staff degli studenti coordinato da Alessandro Gargini. 